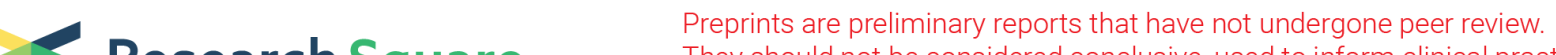 $\begin{array}{ll}\text { Research Square } & \text { They should not be considered conclusive, used to inform clinical practice, } \\ \text { or referenced by the media as validated information. }\end{array}$
}

\section{Antimicrobial peptide AMP-17 exerts anti-Candida albicans effects through ROS-mediated apoptosis and necrosis}

\author{
Huiling Ma
}

Guizhou Medical University

Longbing Yang

Guizhou Medical University

Zhuqing Tian

Guizhou Medical University

Lijuan Zhu

Guizhou Medical University

JiangFan Xiu

Guizhou Medical University

\section{Ping Fu}

Guizhou Medical University

Jian Peng

Guizhou Medical University

Guo Guo ( $\nabla$ guoguojsc@163.com )

Guizhou Medical University

\section{Research article}

Keywords: AMP-17, Candida albican, reactive oxygen species, apoptosis

Posted Date: December 3rd, 2020

DOl: https://doi.org/10.21203/rs.3.rs-119612/v1

License: (1) (1) This work is licensed under a Creative Commons Attribution 4.0 International License. Read Full License 


\section{Abstract}

Background: New anti-candida albicans drugs need to be developed due to the emergence of drugresistant cases in recent years. AMP-17 (Musca. domestica antimicrobial pepitide-17) is an antimicrobial peptide from $M$. domestica, which inhibits many fungal pathogens including Candida albicans $(C$. albicans) effectively. In this article, we discuss the potential mechanism of AMP-17 against $C$. albicans from the perspective of affecting its cell internal structure.

Methods: After AMP-17 treatment, we examined the ultrastructure of $C$. albicans by transmission electron microscopy (TEM) and detected the cell cycle using flow cytometry. Fluorescent probes were used to examine the reactive oxygen species (ROS) accumulation in $C$. albicans cells and to analyze the correlation between ROS accumulation and $C$. albicans cell necrosis. The JC-1 kit was used to measure the effect of AMP-17 on the mitochondrial membrane potential (MMP) of $C$. albicans cells. AMP-17induced apoptosis and necrosis was investigated using an Annexin V-FITC apoptosis detection kit.

Results: Morphological observations showed that the shape of $C$. albicans treated with AMP-17 was irregular, and vacuoles were found in the cytoplasmic region. The treatment of C. albicans with AMP-17 resulted in the elevation of reactive oxygen species (ROS), depolarization of mitochondrial membrane potential (MMP), and changes in cell cycle, which promoted apoptosis and necrosis of $C$. albicans cells. The level of apoptosis increased in a dose-dependent manner after AMP-17 treatment.

Conclusions: AMP-17 inhibited the growth and proliferation of $C$. albicans cells by altering the cell cycle of $C$. albicans. In addition, AMP-17 stimulated mitochondria to produce excess ROS for anti-stress, but the excess ROS damages the function of mitochondria in return and results in the alteration of MMP. All of these ultimately contributes to the death of $C$. albicans.

\section{Introduction}

Candida albicans is an opportunistic pathogen with dimorphic phenotype, including yeast and hyphae [1-2]. Under the conditions of normal immune function, it can survive on the skin, oral cavity, a gastrointestinal and genitourinary tract of healthy people in a commensal way without causing a wide range of infections [3]. When the immune system is damaged, such as AIDS patients, using immunosuppressive agents for organ transplant patients and patients with microbial flora disorders, $C$. albicans that exists in a commensal manner can easily transform into a pathogenic fungal pathogen [4]. Such patients with specific risk factors are generally susceptible to $C$. albicans, and the site of infection may be almost all organs [5]. In recent years, due to the widespread use of traditional antifungal agents such as triazoles, the isolation rate of resistant $C$. albicans strains has been increasing, which has brought great challenges to the treatment of $C$. albicans infection [6-7]. In this context, there is a critical need to develop effective antifungal agents with unique structures and mechanism of action to treat infections caused by Candida species. 
Antimicrobial peptides (AMPs) are a class of small biologically active peptides produced by a variety of organisms as the first line of defense against pathogens. When the hosts are infected or subject to immune stimulation, this kind of small-molecule peptide can be quickly synthesized and in large quantities in some tissues and cells. According to reports in the literature, in addition to inhibiting pathogens such as bacteria and fungi, AMPs also have varying degrees of killing effects on viruses, tumor cells, and parasites [8-9]. Compared to traditional antibiotics, AMPs have a broader spectrum of antimicrobial effects, better thermal stability, and less toxicity to human cells. In addition, they are considered to be the most promising alternatives to traditional antibiotics because they are not easily susceptible to microbial resistance [10]. Since the first discovery of Cecropins in insect blood lymphocytes by Swedish biologist Boman [11], insect AMPs have become a research hotspot in life sciences.

Musca domestica is the most abundant and common Diptera insect and can be easily found in most parts of the world [12]. It usually lives in places where the environment is extremely dirty and easy to carry a large number of pathogens [13].

However, houseflies themselves are not susceptible to infection by pathogenic microorganism, mainly due to their strong innate system. Among them, inducing the expression of AMPs is a very important and effective defense strategy. AMP-17 (Musca domestica antimicrobial peptide-17) is encoded by a specific high-expression gene selected from $M$. domestica transcriptome database constructed after microbial infection 12 hours. In the previous study, our research team successfully produced the recombinant protein AMP-17 with excellent antifungal activity in a prokaryotic expression system [14-15]. Further research has found that it can reduce the cell wall integrity of $C$. albicans, destroy the cell membrane structure, and increase cell membrane permeability [16]. However, whether AMP-17 acts on the intracellular target of $C$. albicans remains unclear. To answer this question, we conducted an in-depth study on the potential anti-Candida mechanism of AMP-17 from the perspective of the influence on the internal structure of $C$. albicans.

An antimicrobial peptide can often act through multiple mechanisms. Studies have shown that simply increasing the permeability of the cell membrane may not be enough to cause the death of pathogens. In addition to changing the permeability of the membrane, AMPs also target the contents of the cell to inhibit transcription, translation or other Biological process [17]. Tian and coworkers showed that the antifungal activity of Perillaldehyde (PAE) against $C$. albicans was correlated with an elevation in intracellular $\mathrm{Ca}^{2+}$ and accumulation of ROS. Several downstream apoptosis events such as the disruption of mitochondrial membrane potential, phosphatidylserine externalization, cytochrome c release, and metacaspase activation were observed in PAE-treated cells [18]. ROS accumulation is considered to be a typical hallmark of apoptosis. Papiliocin, a 37-residue peptide isolated from the genus Papilio Xuthus, can induce mitochondrial membrane damage through the accumulation of ROS, leading to cell dysfunction and ultimately cell apoptosis or necrosis [19]. These previous reports prompted us to determine whether AMP-17 induced the apoptosis through ROS dependent pathway. 
In this study, we examined the ultrastructure of $C$. albicans by transmission electron microscopy (TEM) and detected the cell cycle of $C$. albicans using flow cytometry. To further clarify the mode of antifungal action, fluorescent probes were used to detect the reactive oxygen species (ROS) accumulation in $C$. albicans cells and to analyze the correlation between ROS accumulation and cell necrosis in C. albicans. Besides, the JC-1 kit was used to measure the effect of AMP-17 on intracellular mitochondrial membrane potential (MMP). At last, AMP-17-induced apoptosis was investigated using an Annexin V-FITC apoptosis detection kit. The goal of this research is to explore the target of AMP-17's antifungal effect more deeply, which is the key to enhancing its therapeutic potential.

\section{Materials And Methods Peptides and reagents}

The recombinant AMP-17 protein was obtained with a prokaryotic expression system and purified by nickel ion metal chelator affinity chromatography. The minimal inhibitory concentration (MIC) of recombinant AMP-17 on C. albicans was $20 \mu \mathrm{g} / \mathrm{mL}$ with the micro-liquid dilution method detected.

Propidium iodide (PI), Ribonuclease A (RNAse A), Glutathione (GSH), and 2',7'-dichlorofluorescin diacetate (DCFH-DA) were purchased from Sigma Chemicals (St Louis, MO, USA). Sabouraud dextrose agar (SDA), Sabouraud dextrose Broth (SDB), and ascorbic acid(AA) were purchased from Solarbio (Beijing, China).

\section{Strains and culture conditions}

C. albicans (ATCC10231) was stored in a tube containing $30 \%$ glycerol at $-80{ }^{\circ} \mathrm{C}$ and sub-cultured twice on an SDA plate. Before each experiment, cells were cultured in SDB for $18 \mathrm{~h}$ on a shaker incubator (200 rpm) at $37^{\circ} \mathrm{C}$.

\section{Analysis of cell morphology}

To visualize the effect of AMP-17 on the ultrastructure of $C$. albicans cell, transmission electron microscopy (TEM) was applied. C. albicans cells with an initial density of $1.0 \otimes 5.0 \times 10^{6}$ colony forming units (CFU) $/ \mathrm{mL}$ were treated with $40 \mu \mathrm{g} / \mathrm{mL}$ of AMP- 17 at $37^{\circ} \mathrm{C}$ for $16 \mathrm{~h}$, and then collected by centrifugation (5000 rpm for $10 \mathrm{~min}$ ). Cells without drug treatment served as the control. The samples of each group were washed with phosphate buffer saline (PBS) for 2 times and fixed with $1 \mathrm{ml} 2.5 \%$ glutaraldehyde at $4^{\circ} \mathrm{C}$ overnight. Then, the fixed samples were washed twice with PBS and dehydrated by a sequence of $50 \%, 75 \%$, and $100 \%$ ethanol solution. At last, they were observed under a Hitachi $\mathrm{H}-7650$ TEM (Tokyo, Japan).

\section{Cell cycle analysis using propidium iodide by Flow cytometry}

C. albicans cells were diluted to $1.085 .0 \times 10^{6} \mathrm{CFU} / \mathrm{mL}$ with SDB medium, and AMP-17 at a final concentration of $20 \mu \mathrm{g} / \mathrm{mL}, 40 \mu \mathrm{g} / \mathrm{mL}$, and $80 \mu \mathrm{g} / \mathrm{mL}$ was used as an experimental group. Sterile water 
was the control group. Samples were placed at $37^{\circ} \mathrm{C}$ for $12 \mathrm{~h}$, and the cells were collected by centrifugation at $3000 \mathrm{rpm}$ for 5 min and washed twice with PBS buffer. Resuspend each group of cells with $70 \%$ ethanol solution pre-cooled in advance, fixed at $4{ }^{\circ} \mathrm{C}$ for more than $18 \mathrm{~h}$. The fixed groups of cells were collected by centrifugation at a low temperature of $3000 \mathrm{rpm}$ for $5 \mathrm{~min}$ and washed twice with a sterile PBS buffer. Each group of cells was added with RNaseA, incubated at $37^{\circ} \mathrm{C}$ for $2 \mathrm{~h}$, added PI staining solution ( $\mathrm{Pl}$ concentration of $0.1 \mathrm{mg} / \mathrm{ml}$ ), stained at $4{ }^{\circ} \mathrm{C}$ for $18 \mathrm{~h}$ in the dark, and detected by flow cytometry.

\section{Measurement of ROS formation in C. albicans}

The dye DCFH-DA could freely penetrate cell membranes and be hydrolyzed by lipase to produce nonfluorescent DCFH, which could be rapidly oxidized by intracellular ROS into 2',7'-dichlorofluorescein (DCF) with high fluorescence intensity. So we detect the fluorescence level of DCF to reflect the generation of intracellular ROS [20]. C. albicans cells with an initial density of $1.0 \otimes 5.0 \times 10^{6} \mathrm{CFU} / \mathrm{mL}$ were treated with 0 (control), 20,40 , and $80 \mu \mathrm{g} / \mathrm{mL}$ of AMP- 17 at $37^{\circ} \mathrm{C}$ for $12 \mathrm{~h}$. The positive control sample was incubated with fluconazole (FLC) at the same condition. Then, cells were collected and fixed for DCFH-DA with a final concentration of $20 \mu \mathrm{g} / \mathrm{mL}$. After $30 \mathrm{~min}$ of incubation at $30^{\circ} \mathrm{C}$, the cells were collected, washed twice, and then diluted to $10^{6} \mathrm{CFU} / \mathrm{mL}$ with SDB. The fluorescence intensities (excitation and emission of 485 and $530 \mathrm{~nm}$, respectively) of cells were measured with a microplate reader (Berthold Biotechnologies, Bad Wildbad, Germany) and the fluorescence images were taken using a confocal laser scanning microscopy (CLSM) with FITC filter (Olympus FV1000, Olympus, Tokyo, Japan). To test the effect of antioxidants on ROS accumulation caused by AMP-17, these experiments were also conducted in the presence of $5 \mathrm{mM} \mathrm{AA}, \mathrm{GSH}$ and $50 \mathrm{mM} \mathrm{AA}, \mathrm{GSH}$.

\section{Effect of Antioxidants AA and GSH on antifungal activity of AMP-17}

C. albicans solution in the logarithmic growth phase was diluted to $1 \times 10^{3} \mathrm{CFU} / \mathrm{mL}$ with SDB medium. An aliquot of $100 \mu \mathrm{l}$ of the final suspension was added into each well on a sterile 96-well plate containing $100 \mu \mathrm{l}$ of medium containing AMP-17 at double-diluted concentrations. Candida albicans cells in the experimental group were pretreated with $5 \mathrm{mM} \mathrm{AA}, 5 \mathrm{mM} \mathrm{GSH}, 50 \mathrm{mM} \mathrm{AA}$, and $50 \mathrm{mM} \mathrm{GSH}$ for $30 \mathrm{~min}$. The plate was incubated at $37^{\circ} \mathrm{C}$ for $48 \mathrm{~h}$. The MIC value was determined as the minimal concentration at which no growth of microbes can be observed by naked eyes according to the standard criteria from the US Clinical Laboratory and Standards Institute (CLSI).

Detection of the correlation between AMP-17-induced ROS accumulation and necrosis using double staining of DCFH-DA and PI

C. albicans cells at a concentration of $1.0 \otimes 5.0 \times 10^{6} \mathrm{CFU} / \mathrm{mL}$ were cultured in SDB containing $80 \mu \mathrm{g} / \mathrm{mL}$ AMP- 17 at $37^{\circ} \mathrm{C}$ for $12 \mathrm{~h}$. Sterile water was used as a negative control. The cells were centrifuged for $10 \mathrm{~min}$ at $5000 \mathrm{r} / \mathrm{min}$ and stained with $10 \mu \mathrm{g} / \mathrm{mL}$ DCFH-DA and $20 \mu \mathrm{g} / \mathrm{mL}$ PI and then detected by CLSM with FITC filter for green fluorescence and PI filter for the red after 30 min incubation in dark. 


\section{Measurement of mitochondrial membrane potential (MMP)}

5,5',6,6'-Tetrachloro-1,1',3,3'-tetraethyl-benzimidazolyl carbocyanine iodide (JC-1; Molecular Probes) was used to analyze changes in mitochondrial membrane potential. C. albicans cells $\left(1.0 \otimes 5.0 \times 10^{6} \mathrm{CFU} / \mathrm{mL}\right)$ were treated with 0 (control), 20, 40, and $80 \mu \mathrm{g} / \mathrm{mL}$ of AMP-17 at $37^{\circ} \mathrm{C}$ for $12 \mathrm{~h}$. The positive control sample was incubated with CCCP at the same condition. Then cell suspensions were stained by $5 \mu \mathrm{mol} / \mathrm{L}$ $\mathrm{JC}-1$ at $37^{\circ} \mathrm{C}$ for $30 \mathrm{~min}$ in the dark. The fluorescence of JC-1 (red fluorescence and green fluorescence) was monitored at $\mathrm{Ex} / \mathrm{Em}=490 / 525 \mathrm{~nm}$ and $490 / 590 \mathrm{~nm}$ with a microplate reader (Berthold Biotechnologies, Bad Wildbad, Germany).

\section{Analysis of apoptosis and necrosis induced by AMP-17}

C. albicans cells at a concentration of $1.0 \otimes 5.0 \times 10^{6} \mathrm{CFU} / \mathrm{mL}$ were treated with 0 (control), 20, 40, and $80 \mu \mathrm{g} / \mathrm{mL}$ of AMP-17 and incubated at $37^{\circ} \mathrm{C}$ for $12 \mathrm{~h}$. Then, cells were collected by centrifugation (5000 rpm for $10 \mathrm{~min}$ ) and washed twice in PBS buffer. Each sample was processed according to the instructions of AnnexinV/FITC Kit. The stained cells were observed by CLSM (Olympus FV1000, Olympus, Tokyo, Japan).

\section{Results}

\section{Transmission electron microscopy}

Untreated $C$. albicans cells were normal and intact, with a plump appearance, smooth surface and clear cell boundaries. Cells were uniform in size and showed a round or oval shape (Fig. 1A and D). After treatment with $40 \mu \mathrm{g} / \mathrm{mL}$ AMP-17 for $16 \mathrm{~h}, C$. albicans showed severe irregularities in shape, and some vacuoles were seen at the junction of the cell membrane and cytoplasm. More importantly, the electron density in the cytoplasm region was abnormal. The edge of intracellular organelles was irregular and vacuoles appeared around the nucleus (Fig. 1B, C, E, F).

Effect of AMP-17 on the cell cycle of C. albicans

As shown in Fig. 3, after the addition of AMP-17, the number of $C$. albicans cells in the S phase gradually increased from 30.94-64.63\%, indicating that AMP-17 treatment AMP-17 treatment resulted in more cells accumulating in $S$ phase. The results suggest that most of cells stopped in the $S$ phase and no longer continued to $\mathrm{M}$ phase.

\section{Effect of AMP-17 on ROS production}

ROS is produced under normal physiological conditions and participates in various biological processes. However, excessive ROS production can cause oxidative damage to cells [25]. In this study, the fluorescent probe DCFH-DA was utilized as a ROS indicator to study the effect of AMP-17 on intracellular ROS production. The results of the microplate reader showed that AMP-17 promoted cellular ROS 
generation in a dose-dependent manner. Quantification of cellular ROS generation in AMP-17-treated $C$. albicans cells revealed a significant increase after incubation with $80 \mu \mathrm{g} / \mathrm{ml}$ of AMP-17 for 12 hours, comparable to the fluconazole-treated group (Fig. 3A). Under the view of CLSM, more green fluorescence cells were observed with increasing AMP-17 concentration, indicating the elevated ROS production (Fig. 3C). The effects of antioxidant AA and GSH on AMP-17-induced ROS accumulation in C. albicans were shown in Fig. 3B. Without antioxidant AA and GSH, AMP-17 induced ROS accumulation in $C$. albicans in a dose-dependent manner. However, in the presence of antioxidant AA and GSH, AMP-17induced ROS was significantly inhibited.

\section{The attenuation of antioxidants AA and GSH for the antifungal effect of AMP-17}

To determine whether the ROS production induced by AMP-17 is involved in its antifungal activity, the anti-Candida activity of AMP-17 was examined with or without the supplement of the ROS scavengers, ascorbic acid (AA) and glutathione (GSH). In the control group without antioxidants VC and GSH, the MIC of AMP-17 against $C$. albicans was $20 \mu \mathrm{g} / \mathrm{ml}$; after adding two antioxidants, the activity of AMP-17 against $C$. albicans was significantly reduced. $5 \mathrm{mM}$ AA and GSH increased the MIC values of AMP-17 by 2-fold, from $20 \mu \mathrm{g} / \mathrm{ml}$ to $40 \mu \mathrm{g} / \mathrm{ml} ; 50 \mathrm{mM} \mathrm{AA}$ and GSH increased the MIC of AMP-17 against $C$. albicans by 8 and 4 times, respectively (Fig. 4). These results indicate that the anti-Candida activity of AMP-17 may be mediated by ROS production. In other words, AMP-17 can exert antifungal activity by inducing ROS accumulation.

Correlation between ROS accumulation and necrosis in C. albicans induced by AMP-17

An increased level of ROS within the cellular environment is one of the factors leading to cell necrosis. To elucidate the relationship between cell necrosis and ROS accumulation induced by AMP-17, we examined DCFH-DA (probe for detecting intracellular ROS) and PI (indicator dye for cell necrosis) stained cells by CLSM. After treatment with $80 \mu \mathrm{g} / \mathrm{ml}$ of AMP-17, C. albicans cells stained by PI and DCFH-DA increased significantly compared to the control group (Fig. 5). The results indicate that $C$. albicans cell necrosis induced by AMP-17 is related to the accumulation of intracellular ROS.

\section{Alteration of mitochondrial membrane potential (MMP) induced by AMP-17}

Mitochondria play an important role in maintaining energy metabolism and regulating cell growth, differentiation and death. Stable MMP is essential for normal cellular function [26, 28]. We evaluated the effect of AMP-17 on MMP of $C$. albicans. The results showed that AMP-17 could decrease the MMP of $C$. albicans in a dose-dependent manner. Compared with the control group, AMP-17 at $20 \mu \mathrm{g} / \mathrm{ml}, 40 \mu \mathrm{g} / \mathrm{ml}$, and $80 \mu \mathrm{g} / \mathrm{ml}$ can reduce the MMP to $3.933,3.133$, and 2.767, respectively. The CCCP serves as a positive control, which can reduce the MMP to 2.067 (Fig. 6). These results indicate that AMP-17 affect the mitochondrial physiological integrity leading to its membrane potential depolarization. 


\section{The apoptosis and necrosis of C. albicans induced by AMP-17}

The Annexin V-FITC/PI KIT was applied to confirm the apoptosis and necrosis induced by AMP-17. In normal cells, phosphatidylserine (PS) is present in the inner leaflet of the plasma membrane, but in apoptotic and necrotic cells, PS is exposed on the outer leaflet. PS exposure is one of the typical manifestations of early apoptotic events. Annexin V-FITC binds to the exposed PS and produces green fluorescence under $488 \mathrm{~nm}$ laser excitation. PI penetrates the damaged cell membrane and enters the cell to combine with the nucleus and generates red fluorescence under $555 \mathrm{~nm}$ laser excitation. Confocal microscopy showed that the green fluorescence and the red fluorescence seen in 12-h, AMP-17-treated $C$. albicans cells showed a gradual increase with the increasing concentration of AMP-17 (Fig. 7), suggesting that AMP-17 induced $C$. albicans apoptosis and necrosis.

\section{Discussion}

Candida albicans, with yeast and mycelium growth forms, is the most common isolated fungus from bloodstream infections. Although significant progress has been made in diagnosis and treatment, these infections continue to be a serious challenge for ICUs (intensive care units) worldwide. The yeast state of C. albicans with unique structure and characteristics is more likely to spread in the blood circulation of debilitated patients $[7,20]$. Based on this diffusion property, inhibition of the proliferation of $C$. albicans yeast is an effective antifungal method. It has been reported in the literature that the proliferation rate of C. albicans is closely related to the cell cycle, so the development of drugs that can prolong the cell cycle has become an effective strategy for the treatment of Candida infections [22]. This experiment found that AMP-17 can block the cell cycle of $C$. albicans in S phase. S phase is the most important stage in the cell cycle process, in which cells undergo DNA replication, synthesize histones and non-histones, and finally complete chromosome replication. If the cells are arrested in the $S$ phase, it can inhibit DNA synthesis, hinder the cells from entering the $\mathrm{M}$ phase, and limit their further growth.

Reactive oxygen species (ROS) are by-products of cellular metabolism mainly present in mitochondria. When $C$. albicans cells encounter external stimuli such as oxidants, heat shocks, metal ions and other factors, cells enhance ROS generation to resist the pressure of the external environment $[20,23,24]$. If the ROS produced by the cell exceeds its metabolic capacity, the excess intracellular ROS induces oxidative damage to lipids, DNA, proteins and other cellular components (such as plasma membrane). The induction of these processes is related to cellular death caused by apoptosis or necrosis [25-26]. This study found that AMP-17 treatment increased the ROS generation and showed a good concentrationdependent effect, but when the $C$. albicans cells were pretreated with two antioxidants, glutathione, and ascorbic acid, the accumulation of intracellular ROS induced by the AMP-17 in C. albicans was significantly inhibited. To further verify that ROS accumulation is a stress response of cells to changes in the external environment, or is the source of AMP-17's antifungal activity, we examined the effect of antioxidants on the activity of the AMP-17. It was found that the antioxidants AA and GSH reduced the antifungal activity of AMP-17 against $C$. albicans. In other words, AMP-17 exert antifungal activity by inducing ROS accumulation. 
As mentioned earlier, the intracellular ROS accumulation leads to fatal consequences such as necrosis, apoptosis and oxidative stress. Necrosis is a form of pathological cell death induced by extreme physical, chemical or other serious pathological stimuli $[25,27,28]$. In our study, using DCFH-DA and PI double staining method proved that the cell necrosis induced by AMP-17 is associated with the increase of intracellular ROS production. Moreover, the damage in cellular membrane integrity is one of the hallmark events of cell necrosis [25]. In previous studies, our experimental data have demonstrated that AMP-17 can cause the loss of integrity of $C$. albicans cell membrane by destroying the membrane structure and increasing the permeability of plasma membrane [16]. In addition, abnormal vacuoles in the cytoplasm was clearly visualized by TEM study, which is also one of the typical features of cell necrosis. These results all reveal the important role of AMP-17 in C. albicans cell necrosis.

Mitochondria are the organelles of eukaryotic cells that produce energy, and are also considered as a major site of ROS generation, so it is both the origin and the target of ROS [29-30]. When the accumulation of ROS in the cells is excessive or the antioxidant defense system is weakened, free radicals in the mitochondria cannot be effectively eliminated. Excess ROS can oxidize the corresponding redox-sensitive sites on the mitochondrial permeability transport pores, leading to mitochondrial damage [31]. In addition, the accumulation of ROS causes lipid peroxidation, which destroys the performance of mitochondrial membranes and triggers changes in membrane potential [32]. In this experiment, the mitochondrial membrane potential (MMP) of $C$. albicans cells was detected by $\mathrm{JC}-1$ fluorescent reagent. It was found that the MMP level gradually decreased after AMP-17 treatment, and $80 \mu \mathrm{g} / \mathrm{ml}$ AMP-17 caused a significant depolarization of the MMP.

Extensive studies suggest that maintenance of mitochondrial membrane potential is a prerequisite for normal mitochondrial function[25,33,34]. Depolarization of MMP is considered to be the earliest event in apoptotic processes. Apoptosis is a form of programmed cell death, which is vital for organisms homeostasis and maintenance by eliminating damaged, unwanted, mutated and redundant cells[26, 35 , 36]. In addition to multicellular organisms, apoptosis also exists in unicellular organisms such as bacteria and yeast[37-38]. In this study, we used AnnexinV-FITC and PI co-staining method to detect whether AMP-17 can cause $C$. albicans apoptosis and necrosis. The results showed that AMP-17-treated $C$. albicans cells exhibited PS exposure and cell membrane damage indicating that AMP-17 induced apoptosis and necrosis, which could be the reason of mitochondrial dysfunction and ROS accumulation.

\section{Conclusions}

In the present study, AMP-17 inhibits the growth and proliferation of $C$. albicans cells by altering the cell cycle. Further research shows that AMP-17 notably increase the production of intracellular ROS in a dosedependent manner and excessive ROS causes oxidative damage to the mitochondrial and other cellular components. Mitochondrial dysfunction and ROS accumulation are the main reasons that trigger cell apoptosis and necrosis. To summarise, AMP-17 exerts its anti- $C$. albicans effect in a variety of ways. The multiple targets of AMP-17 in C. albicans indicate that it may be a new treatment option for the prevention and control of clinical fungal infections. 


\section{Abbreviations}

AMPs: antimicrobial peptides; MIC:minimum inhibitory concentration; SDB:Sabouraud dextrose broth; CLSI:Clinical and Laboratory Standards Association; ROS:Reactive oxygen species; MMP:mitochondrial membrane potential; TEM:Transmission electron microscopy; CLSM:Confocal laser scanning microscopy.

\section{Declarations}

\section{Acknowledgements}

We are grateful for the support of scientific research equipment from the School of Basic Medicine of Guizhou Medical University.

\section{Authors' contributions}

HM, JP and GG conceived and designed the experiments. HM, LY, ZT and LZ performed the experiments. HM, JP, JX and PF analyzed the data. JP and LY contributed reagents/ materials/ analysis tools. HM and GG wrote the paper. All authors read and approved the final manuscript.

\section{Funding}

This study was supported by National Natural Science Foundation of China [grant numbers: 81760647]. Funders had no role in study design, data collection or analysis, preparation of the manuscript or the decision to publish it.

\section{Availability of data and materials}

The datasets supporting the conclusions of this article are included within the article and its additional files. The raw datasets are available from the corresponding author upon reasonable request.

\section{Ethics approval and consent to participate}

All materials used in this study were approved for use by the Institutional Review Board, and all methods/experiments were conducted in accordance with the guidelines approved by the Ethics Committee of Guizhou Medical University, China.

\section{Consent for publication}

Not applicable.

\section{Competing interests}

The authors report no conflicts of interest in this work.

\section{References}


1.Seth, Rachna, Xess, Immaculata, Jana, Manisha. Diagnosis of Invasive Fungal Infections in Children. Indian Pediatr. 2019;56:229-236.

2.Bertolini M, Ranjan A, Thompson A, Diaz PI, Sobue T, Maas K, et al. Candida albicans induces mucosal bacterial dysbiosis that promotes invasive infection. PLoS Pathog. 2019;15(4).

3.Wiederhold NP. Antifungal resistance: current trends and future strategies to combat. Infect Drug Resist. 2017;10:249-259.

4.Cronin S, Spitzer M, Robbins N, Wright GD. Combinatorial strategies for combating invasive fungal infections. Virulence. 2017;8:169-185.

5.Boniche C, Rossi SA, Kischkel B, Barbalho FV, Moura ÁND, Nosanchuk JD, et al. Immunotherapy against systemic fungal infections based on monoclonal antibodies. J Fungi (Basel). 2020;6:31.

6.Kolve $H$, Ahlke E, Fegeler W, et al. Safety, tolerance and outcome of treatment with liposomal amphotericin $B$ in paediatric patients with cancer or undergoing haematopoietic stem cell transplantation. J Antimicrob Chemother. 2009; 64:383-87.

7.Costa-de-Oliveira, Rodrigues AG. Candida albicans antifungal resistance and tolerance in bloodstream infections: the triad Yeast-Host-Antifungal. Microorganisms. 2020;8:154.

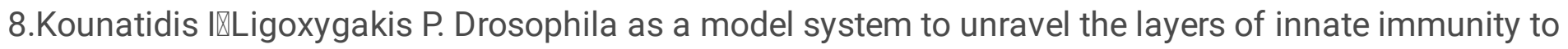
infection. Open Biology. 2012;2:120075.

9.Falanga A, Lombardi L, Franci G, et al. Marine antimicrobial peptides: nature provides templates for the design of novel compounds against pathogenic bacteria. Int J Mol Sci. 2016;17:E785.

10.Sheehan G, Garvey A, Croke M, Kavanagh K. Innate humoral immune defences in mammals and insects: The same, with differences ?. Virulence. 2018;9:1625-39.

11.Boman HG, Nilsson-Faye I, Paul K. Insect immunity i. characteristics of an inducible cell-free antibacterial reaction in hemolymph of Samia cynthia pupae. Infect Immun. 1974;10:136-45.

12. Hou L, Shi Y, Zhai P, Le G. Antibacterial activity and in vitro anti-tumor activity of the extract of the larvae of the housefy (Musca domestica). J Ethnopharmacol. 2007;111:227-31.

13.Gremillion KJ, Piperno DR. Human behavioral ecology, phenotypic (developmental) plasticity, and agricultural origins: insights from the emerging evolutionary synthesis. Curr Anthropol. 2009;50:615-9

14.Jiangfan X, Tao W, Yu W, et al. Histological Observation and Expression Patterns of antimicrobial peptides during Fungal Infection in Musca domestica (Diptera: Muscidae) Larvae. Braz Arch Biol Techn. 2016;59. 
15.Guo G, Tao R, Li Y, Ma H, Xiu J, Fu P, Wu J. Identification and characterization of a novel antimicrobial protein from the housefly Musca domestica. Biochem Biophys Res Commun. 2017;490:746-52.

16.Ma H, Zhao X, Yang L, Su P, Fu P, Peng J, et al. Antimicrobial peptide AMP-17 affects Candida albicans by disrupting its cell wall and cell membrane integrity. Infect Drug Resist. 2020;13:2509-20.

17.Lan Y, Ye Y, Kozlowska J, et al. Structural contributions to the intracellular targeting strategies of antimicrobial peptides. Biochim Biophys Acta. 2010;1798: 1934-43.

18.Tian H, Qu S, Wang Y, et al. Calcium and oxidative stress mediate perillaldehyde-induced apoptosis in Candida albicans. Appl Microbiol Biot, 2017;101:3.

19.Hwang B, Hwang J S, Lee J, et al. Induction of yeast apoptosis by an antimicrobial peptide, Papiliocin. Biochem Biophys Res Commun. 2011;408:89-93.

20.Chang $W Q, W u X Z$, Cheng $A X$, et al. Retigeric acid B exerts antifungal effect through enhanced reactive oxygen species and decreased cAMP. Biochim Biophys Acta. 2011;1810:573-76.

21.Li Y, Chang W, Zhang M, Li X, Jiao Y, Lou H. Diorcinol D exerts fungicidal action against Candida albicans through cytoplasm membrane destruction and ROS accumulation. PloS One. 2015;10(6):1-16.

22.Singh A, Sharma S, Khuller G K. cAMP regulates vegetative growth and cell cycle in Candida albicans. Mol Cell Biochem. 2007;304(1-2):331-341.

23.Ding Y , Li Z , Li Y , et al. HSAF-induced antifungal effects in Candida albicans through ROS-mediated apoptosis. RSC Adv. 2016;10:1-8.

24.Golstein P, Aubry L, Levraud JP. Cell-death alternative model organisms: why and which? Rev Mol Cell Biol. 2003;4: 798-807.

25. Haque F, Verma N K, Alfatah M , et al. Sophorolipid exhibits antifungal activity by ROS mediated endoplasmic reticulum stress and mitochondrial dysfunction pathways in Candida albicans. RSC Adv. 2019;9:41639-48

26.Jia C, Zhang J, Yu L, et al. Antifungal activity of Coumarin against Candida albicans is related to apoptosis. Front Cell Infect Microbiol. 2019;8:445.

27.Jamieson D J . Oxidative stress responses of Saccharomyces cerevisiae. Yeast. 1998;14(16):1511-27.

28. Hao B, Cheng S, Clancy CJ, Nguyen MH. Caspofungin kills Candida albicans by causing both cellular apoptosis and necrosis. Antimicrob Agents Ch. 2013;57:326-32.

29.Nunnari J, Suomalainen A. Mitochondria: in sickness and in health. Cell. 2012;148:1145-59. 
30.Terman A, Gustafsson B, and Brunk UT. The lysosomal-mitochondrial axis theory of postmitotic aging and cell death. Chem Biol Interact. 2006; 163:29-37.

31.Pereira C, Silva R, Saraiva L, Johansson B, Sousa M and Côrte-Real M.

Mitochondria-dependent apoptosis in yeast. Biochim Biophys Acta. 2008; 1783:1286-1302.

32.Curtin JF, Donovan M, Cotter TG. Regulation and measurement of oxidative stress in apoptosis. J Immunol Methods. 2002;265: 49-72.

33.Von der HT, Leadsham J E, Sauvadet A, et al. The control of translational accuracy is a determinant of healthy ageing in yeast. Open Biol. 2017; 7:16-29.

34.Sharon A, Finkelstein A, Shlezinger N, et al. Fungal apoptosis: function, genes and gene function. FEMS Microbiol Rev. 2009;33: 833-54.

35.Rockenfeller P and Madeo F. Apoptotic death of ageing yeast. Exp Gerontol. 2008;43:876-81.

36.Shirtliff ME, Krom BP, Meijering RAM, et al. Farnesol-induced apoptosis in Candida albicans. J Antimicrob Chemother. 2009;53: 2392-2401.

37.Arshad A, Osman H, Bagley M, Lam C, Mohamad S and Zahariluddin A. Synthesis and antimicrobial properties of some new thiazolyl coumarin derivatives. Eur J Med Chem. 2011;46:3788-94.

38.Eisenberg T, Büttner S, Kroemer G, et al. The mitochondrial pathway in yeast apoptosis. Apoptosis, 2007;12: 1011-23.

\section{Tables}

Table 1 Percentage of DNA content of C. albicans after treatment with AMP-17 (\%)

\begin{tabular}{|c|c|c|c|}
\hline \multirow{2}{*}{$\begin{array}{l}\text { AMP-17 } \\
\varangle \mu \mathrm{g} / \mathrm{ml}\end{array}$} & \multicolumn{3}{|c|}{ Cell cycle ratio (\%) } \\
\hline & G0/G1 & S & $\mathrm{G} 2 / \mathrm{M}$ \\
\hline 0 & 33.75 & 30.94 & 32.91 \\
\hline 20 & 5.04 & 54.76 & 38.51 \\
\hline 40 & 27.72 & 65.70 & 6.59 \\
\hline 80 & 4.79 & 64.63 & 30.57 \\
\hline
\end{tabular}

\section{Figures}



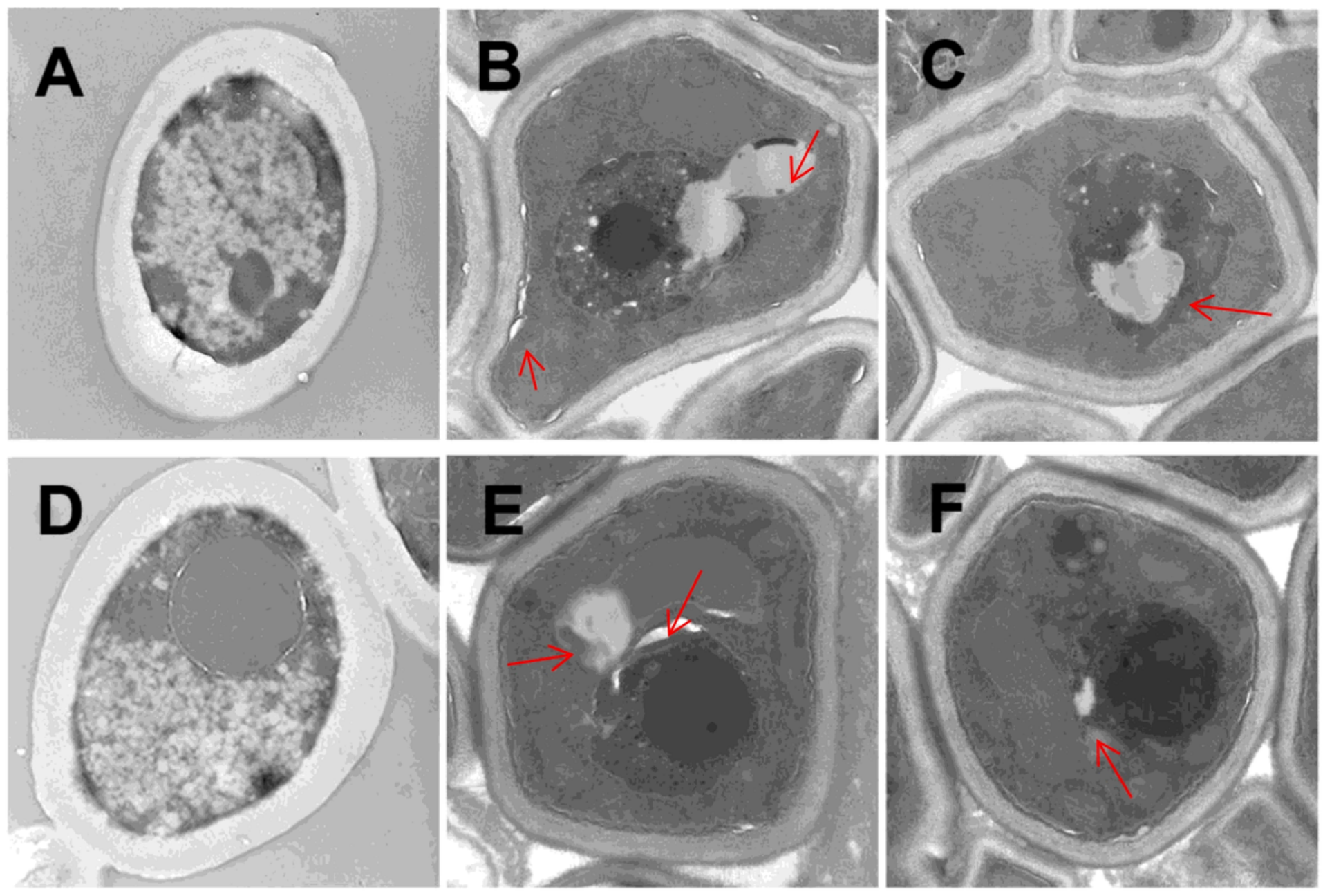

\section{Figure 1}

TEM images of the effect of AMP-17 on the internal structure of C. albicans. (A 40000x, D 50000x) Untreated $C$. albicans cells are intact, with a plump appearance, clear cell boundaries, and normal electron density in the cytoplasm. (B, C 40000x and E, F 50000x) C. albicans treated with AMP-17 $(40 \mu \mathrm{g} / \mathrm{mL}$ ) at $37^{\circ} \mathrm{C}$ for $16 \mathrm{~h}$ showed severe irregularities in shape, and cells adhered tightly to each other. More importantly, abnormal vacuoles of different sizes appeared in the cytoplasmic region of the diseased cells (red arrowheads). 

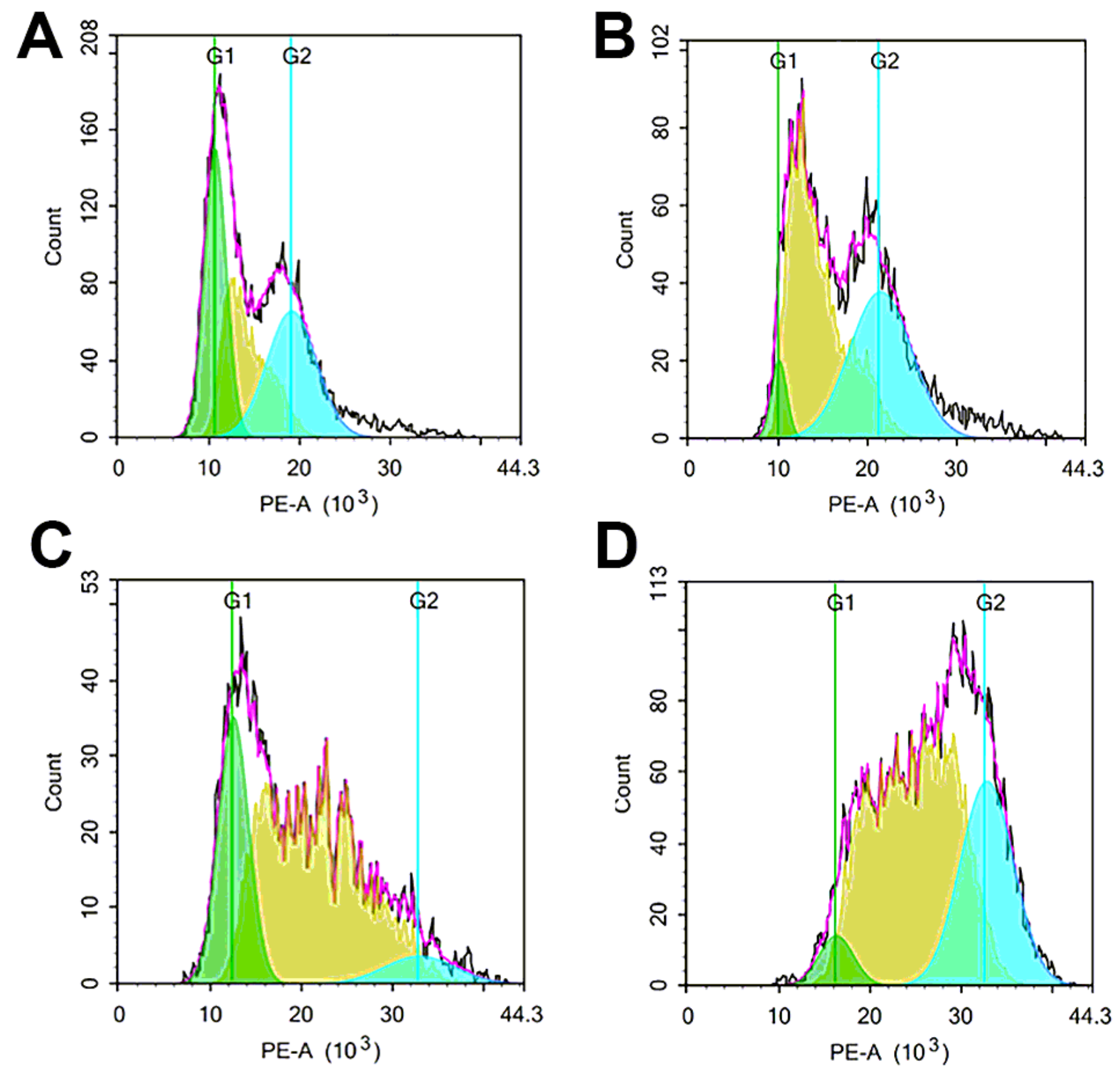

Figure 2

Cell cycle analysis of C. albicans assayed by flow cytometry. (A) Untreated C. albicans cells. (B, C, D) C. albicans cells were incubated with 20,40 , and $80 \mu \mathrm{g} / \mathrm{mL} \mathrm{AMP-} 17$ at $37^{\circ} \mathrm{C}$ for $12 \mathrm{~h}$. 
A $\quad$ B
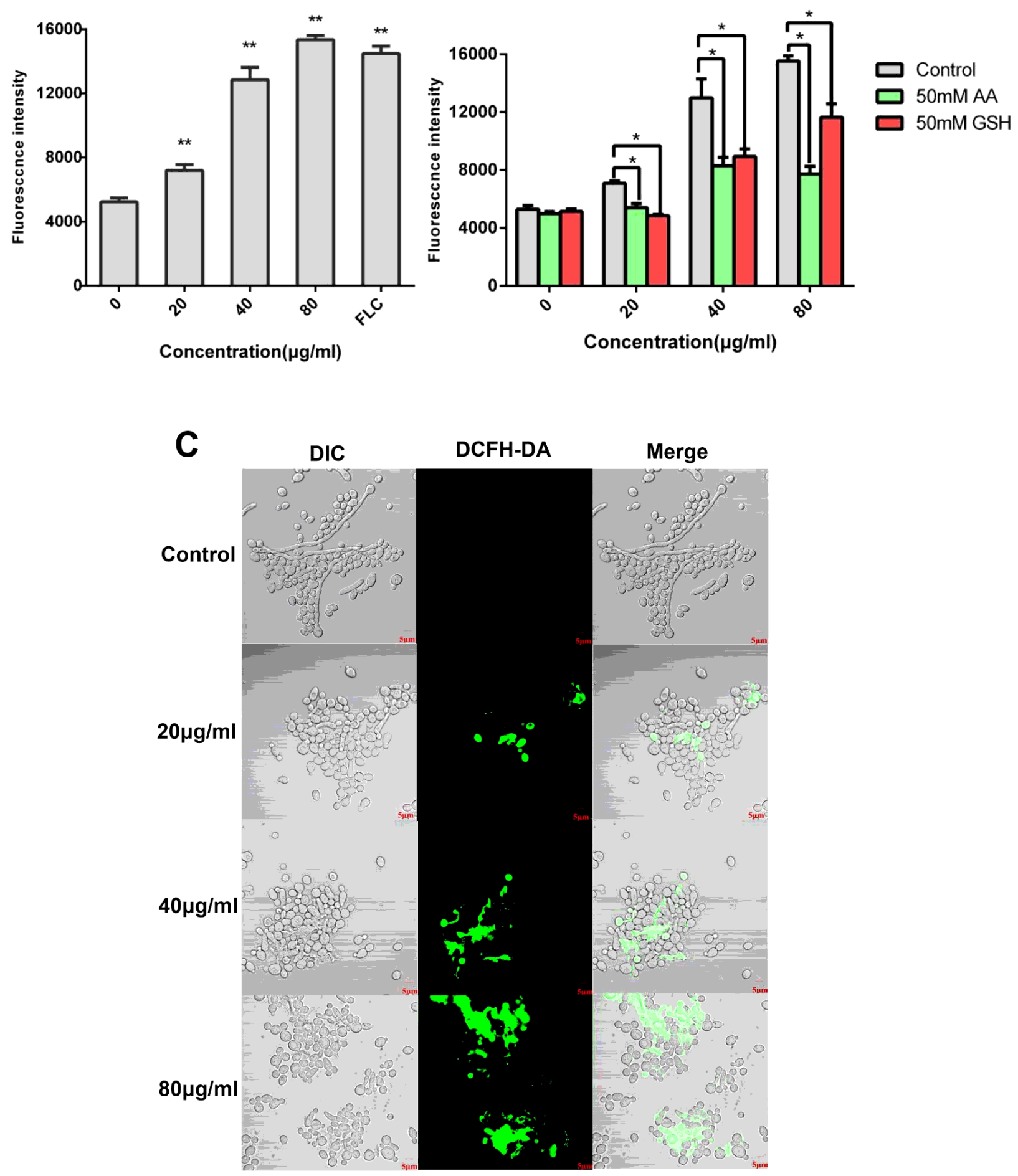

\section{Figure 3}

Effect of AMP-17 on intracellular ROS formation in C. albicans. Cells were incubated with 0 (control), 20, 40 , and $80 \mu \mathrm{g} / \mathrm{mL}$ AMP- 17 at $37^{\circ} \mathrm{C}$ for $12 \mathrm{~h}$. After staining with $20 \mu \mathrm{g} / \mathrm{mL}$ DCFH-DA, the fluorescence in samples was detected by a microplate reader $(A)$ and confocal laser scanning microscopy (C), respectively. Effect of antioxidants AA and GSH on AMP17-induced ROS generation (B). The bars in (A, B) 
indicate standard deviations and in (C) indicate $5 \mu \mathrm{m}$. ${ }^{*}$ Compared with Negative control Pख0.05; ${ }^{* *}$ Compared with Negative control Pख0.01.

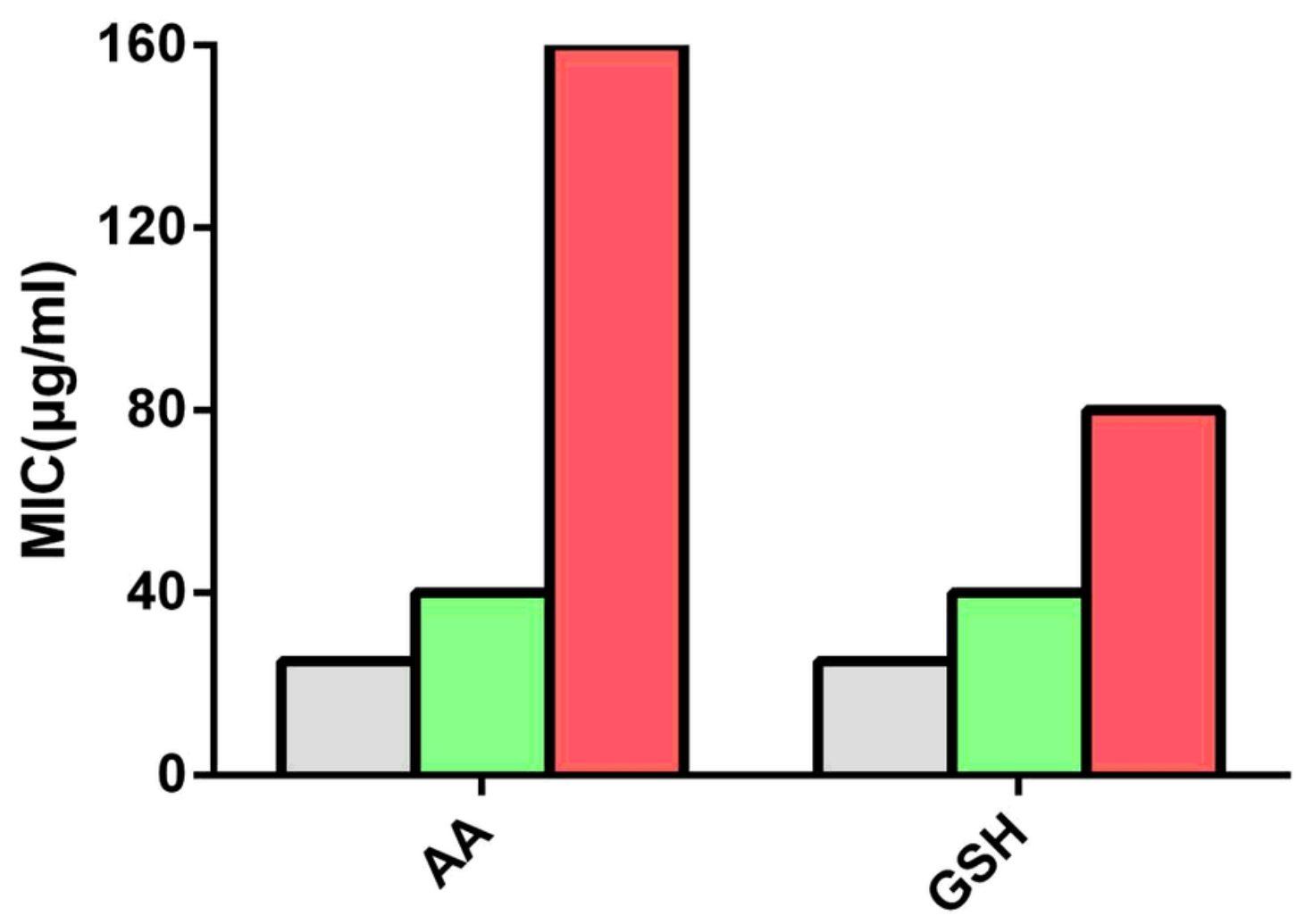

Figure 4

Effect of antioxidants on antifungal activity of AMP-17. C. albicans were incubated with different concentrations of AMP- 17 for $24 \mathrm{~h}$ at $37^{\circ} \mathrm{C}$, meanwhile adding $\mathrm{AA}$ and $\mathrm{GSH}$ for $30 \mathrm{~min}$ prior to the addition of AMP-17. Minimum inhibitory concentration was detected after drug exposure in the presence and absence of AA or GSH. Without the presence of antioxidants VC and GSH, the MIC of AMP-17 against C. albicans is $20 \mu \mathrm{g} / \mathrm{ml}$. After adding two antioxidants, the activity of AMP-17 against C. albicans was significantly reduced. 


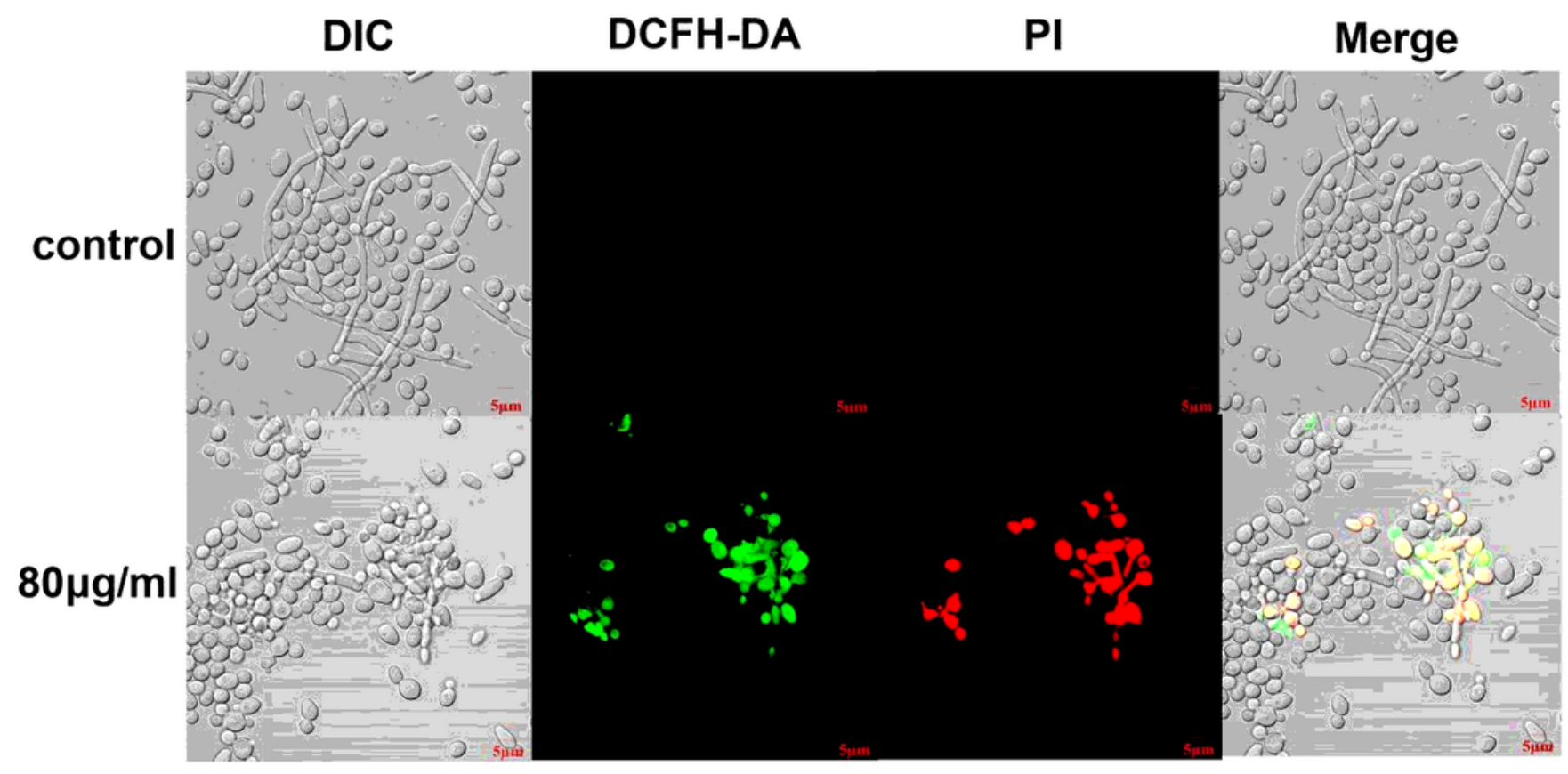

\section{Figure 5}

The correlation between ROS accumulation and necrosis (1000x). C. albicans cells was incubated with 0 (control)and $80 \mu \mathrm{g} / \mathrm{ml}$ of AMP- 17 at $37^{\circ} \mathrm{C}$ for $12 \mathrm{~h}$. After the samples were collected, they were stained with DCFH-DA and PI and detected by confocal laser scanning microscopy after 30 min incubation in dark. The bars indicate $5 \mu \mathrm{m}$. 


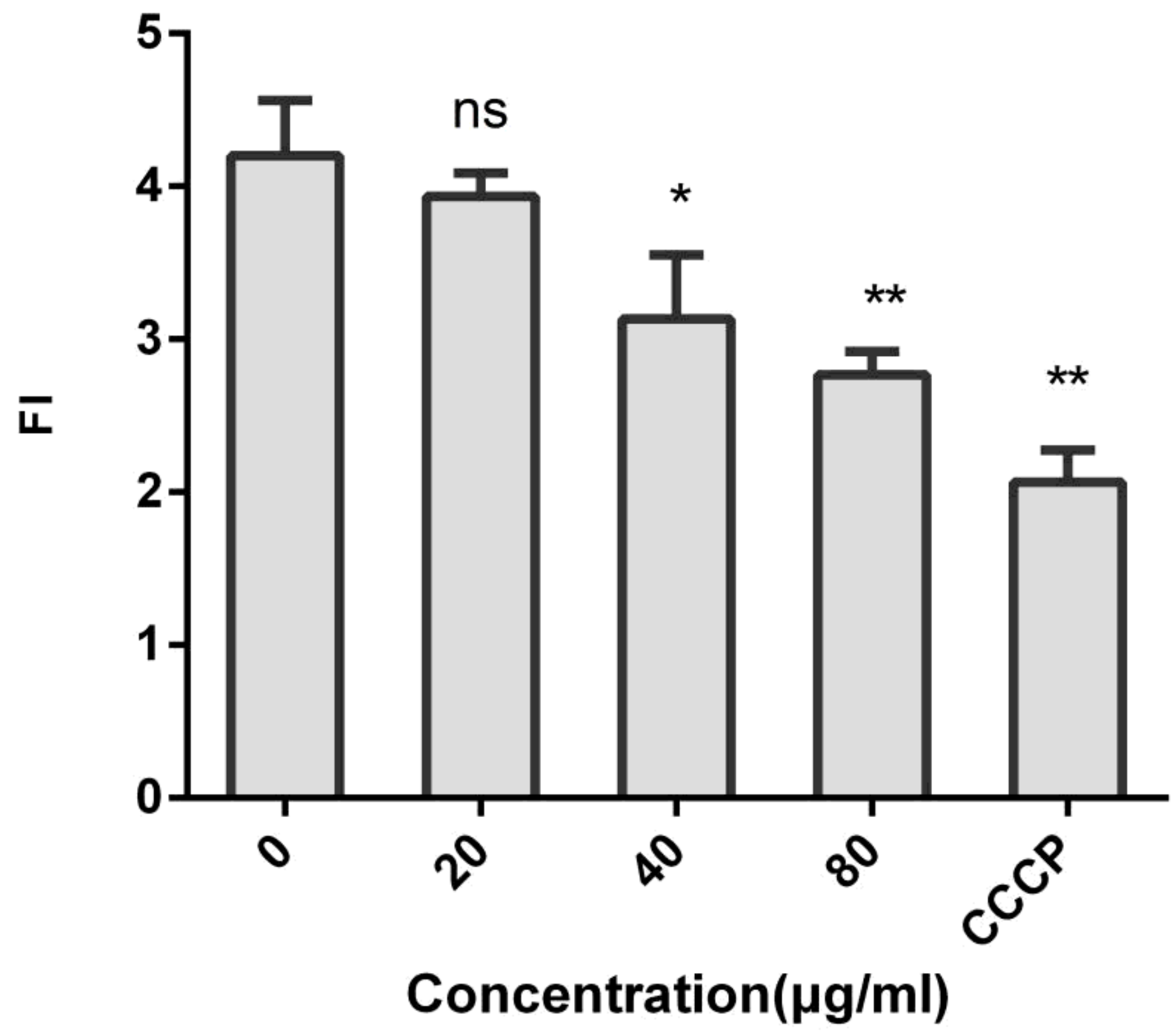

Figure 6

The effect of AMP-17 on the mitochondrial membrane potential of $\mathrm{C}$. albicans cells. Cells were treated with various concentrations of AMP-17 or CCCP (positive control) for $12 \mathrm{~h}$ following with JC-1 staining for spectrofluorophotometric detection. Bars indicate the standard deviations. ${ }^{*}$ Compared with Negative control $\mathrm{P} \otimes 0.05 ;{ }^{* *}$ Compared with Negative control $\mathrm{P} \otimes 0.01$. 


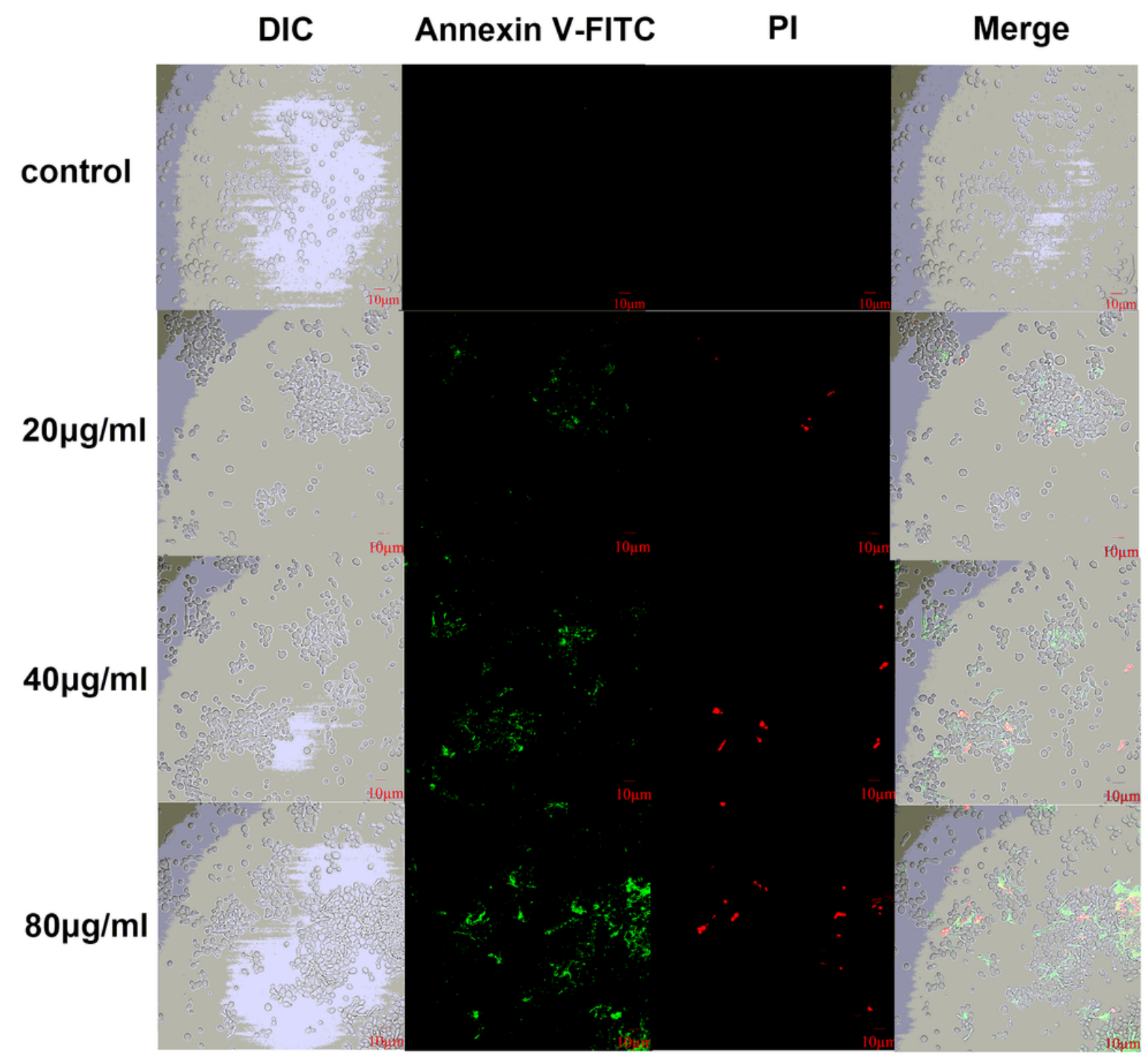

\section{Figure 7}

Observation of AMP-17-induced early apoptosis of C. albicans with confocal laser scanning microscopy (600x). Cells treated with $0,20,40$ and $80 \mu \mathrm{g} / \mathrm{mL}$ AMP-17 at $12 \mathrm{~h}$ shown by AnnexinV-FITC/PI staining. The FITC and PI were excited by $488 \mathrm{~nm}$ and $555 \mathrm{~nm}$ lasers respectively. The bars indicate $10 \mu \mathrm{m}$. 